\title{
Design of soft-X-ray tomographic system in WEST using GEM detectors
}

\author{
Didier Mazon $^{\mathrm{a}}$, Maryna Chernyshova ${ }^{\mathrm{b}}$, Guillaume Jiolat ${ }^{\mathrm{a}}$, Tomasz Czarski ${ }^{\mathrm{b}}$, Philippe Malard ${ }^{\mathrm{a}}$, Ewa \\ Kowalska-Strzeciwilk ${ }^{\mathrm{b}}$, Slawomir Jablonski ${ }^{\mathrm{b}}$, Waldemar Figacz ${ }^{\mathrm{b}}$, Roman Zagorski ${ }^{\mathrm{b}}$, Monika \\ Kubkowska ${ }^{\mathrm{b}}$, Grzegorz Kasprowicz ${ }^{\mathrm{c}}$, Krzysztof Pozniak ${ }^{\mathrm{c}}$, Wojciech Zabolotny ${ }^{\mathrm{c}}$, Sébastien Larroque ${ }^{\mathrm{a}}$, \\ Jean-Marc Verger ${ }^{\mathrm{a}}$, Martin O’Mullane ${ }^{\mathrm{d}}$, Jan Mlynar ${ }^{\mathrm{e}}$, Adrian Byszuk ${ }^{\mathrm{c}}$, Andrzej Wojenski ${ }^{\mathrm{c}}$ \\ ${ }^{a}$ CEA, IRFM F-13108 Saint-Paul-lez-Durance, France. \\ ${ }^{b}$ Institute of Plasma Physics and Laser Microfusion, 23 Hery Street, 01-497 Warsaw, Poland \\ ${ }^{c}$ Warsaw Uniwersity of Technology, Institute of Electronic Systems, Nowowiejska 15/19, 00-665 Warsaw, Poland \\ ${ }^{d}$ Department of Physics, University of Strathclyde, 107 Rottenrow, Glasgow G4 ONG, UK \\ ${ }^{e}$ Institute of Plasma Physics AS CR, Association EURATOM-IPP.CR, Za Slovankou 3, 18200 Prague, Czech Republic
}

\begin{abstract}
In metallic tokamaks, the interplay between particle transport and MagnetoHydroDynamic (MHD) activity might lead to impurities accumulation and finally to disruption. Studying such phenomena is thus essential if stationary discharges are to be achieved. Measuring the Soft X-Ray (SXR) radiation ([0.1 keV; $20 \mathrm{keV}])$ of magnetic fusion plasmas is a standard way of accessing valuable information on particle transport and MHD. Generally, like at Tore Supra (TS), the analysis is performed with a 2D tomographic system composed of several cameras equipped with Silicon Barrier Diodes (SBD). On WEST the installation of an upper divertor masks many of the actual TS vertical diodes so that no proper tomography is possible. This paper presents the design of a new SXR diagnostic for the WEST project developed in collaboration with IPPLM (Poland) and the Warsaw University of Technology, based on a triple Gas Electron Multiplier (GEM) detector. Preliminary simulations performed to size and position the detector and its electronics inside the vertical thimble are also presented, in particular estimation of magnetic field and temperature variation affecting GEM spatial resolution and signal quality. As a conclusion, perspectives about tomographic capabilities of the new system for studying impurity transport are given.
\end{abstract}

Keywords: Soft-X-ray, Gas detector, Tomography, WEST, tokamak

\section{Introduction}

\subsection{Motivations}

Measuring Soft X-Ray (SXR) radiation (0.1-20 $\mathrm{keV}$ ) of magnetic fusion plasmas is a standard way of accessing valuable information on particle transport and magnetic configuration. An increased attention to tungsten material is related to a fact that it became a main candidate for the plasma facing material in ITER and future fusion reactor. Since recently the metallic walls have been installed on many machines and a new problem occurs with the impurities which are released from the wall (W in particular), it is highly desirable, then, to understand the impact of the plasma contamination and its effects on plasma scenarios. As such impurities could cause radiative collapses and often disruption one needs to develop a new diagnostic to be able to reconstruct the impurity distribution. For this purpose a SXR tomographic diagnostic with energy discrimination is a very good candidate [1]. Indeed, SXR radiation depends mainly on electron and impurity densities and on electron temperature, making it information-rich but difficult to analyze. Generally in tokamaks, the SXR analysis is performed with a $2 \mathrm{D}$ tomographic system composed of several cameras equipped with Silicon Barrier Diodes (SBD) but without any energy discrimination capabilities (which means that the full spectrum is integrated). This principle has been utilized for example on Tore Supra (TS): 82 SXR detectors, spread in one horizontal and one vertical camera, were installed for performing the tomographic inversion [2] leading to a spatial resolution of about $4 \mathrm{~cm}$ in the equatorial plane. Presently TS is being under heavy reconstruction for the WEST project and, respectively, the tomographic system will need to be also adapted. The WEST Project aims at implementing an actively cooled tungsten divertor, similar to ITER divertor technology, into the long pulse tokamak TS. One of the main operational difficulties in metallic tokamaks (or with metallic parts like in WEST project) is the interplay between particle transport and MHD activity which might lead to impurities accumulation and finally to disruption. Studying such phenomena is thus essential if stationary discharges are to be achieved. On top of this on WEST the installation of an upper divertor masks many of the actual TS vertical diodes so that no proper tomography is possible. An efficient associated tomographic system is therefore required. 


\subsection{Requirements and constraints}

In order to determine which kind of detector should be selected for the new tomographic system we have first to estimate the physics requirements in WEST. Indeed, as mentioned in the introduction the system is dedicated to the study of impurity transport (in particular W) and MHD activity. In terms of time resolution, impurity transport is rather slow $(1 \mathrm{kHz})$ while MHD modes activity can be rather fast, from $10 \mathrm{kHz}$ (tearing modes, sawtooth crashes) up to $100 \mathrm{kHz}$ (toroidal Alfven eigenmodes). In terms of spatial resolution we estimated that a resolution of $1 \mathrm{~cm}$ in the equatorial plane should be sufficient for getting accurate information about the magnetic axis, the sawteeth inversion radius, the impurity gradients distribution and more generally to get a good tomographic image. This new spatial resolution of $1 \mathrm{~cm}$ has to be compared with the former one used for TS discharges which was of $4 \mathrm{~cm}$ and was already accurate enough to provide nice impurity transport analysis. Based on former work [1], it is requested to get a spectral discrimination of the signal, which means to have a system capable of doing photon counting for several energy bands at a fast rate. The design of the position and energy sensitive X-ray detector for tokamak plasma, should be thus driven by the following requirements: large detection area matching the aperture of the single window of the spectrometer, good spatial resolution, high charge gain capability, detection stability for a wide range of photon rates, energy resolution capability (20-30\%) as well as sustainability to neutron radiation. Finally we have also to take into account geometrical considerations. Indeed, due to the presence of an upper divertor, the vertical camera should be inserted inside the vertical port, if we want to keep tomographic capabilities and see correctly the plasma volume. This cylindrical space is rather narrow $(20 \mathrm{~cm}$ width) and the detector should be rather compact (including its front end analogue electronics).

\section{GEM detectors}

\subsection{Principle and capabilities}

Based on the previous requirement we have selected the GEM technology for the new WEST SXR tomographic system. It has been demonstrated that the GEM technique, when configured in multistep structure, named triple GEM, can provide sufficient detection characteristics [3-5]. This detector works in photon counting mode with energy discrimination, is compact, has good temporal and spatial resolution, and has the particularity to be more neutron resilient than present SXR diodes what makes it a potential good candidate for SXR measurement in ITER and/or DEMO. In general GEM detectors are used for imaging purposes and not tomographic detection in single photon counting mode, making this project strongly innovative. The developed detector is a closed volume, with applied high voltage to its elements, filled with a flowing gas mixture adapted to photoionization by SXR and closed by about $2 \times 20 \mathrm{~cm}^{2}$ Mylar foil. As depicted in Fig. 1, photoelectrons are produced in the first conversion layer and drift towards the first micrometrically perforated copper-clad Kapton foil where electron avalanching amplifies the signal. The detector gain depends on the high voltage used for avalanching. A similar process occurs two more times at successive GEM foils and the resulting charge is then collected on anode strips (pixels).

Up to now the current SXR Si diodes unit was limited in the number of tomographic lines to perform the general tomographic reconstruction. Installation of two 1D GEM based detectors would bring new possibilities of plasma tomography. New system would cover up to 128 viewing lines (if applicable) in vertical and horizontal directions to precisely reconstruct plasma poloidal profile. To obtain good statistics in experimental X-ray spectra, high quantum detection efficiency is required for SXR diagnostics. Because of the energy discrimination requirement, detectors should operate in the proportional mode with relatively low gas gain $\left(\sim 10^{3}\right)$ at high dynamic range to prevent discharges and space-charge saturation. Therefore the front-end analogue electronics for the signal processing should handle signals corresponding to about $10^{4}-10^{5}$ electrons.

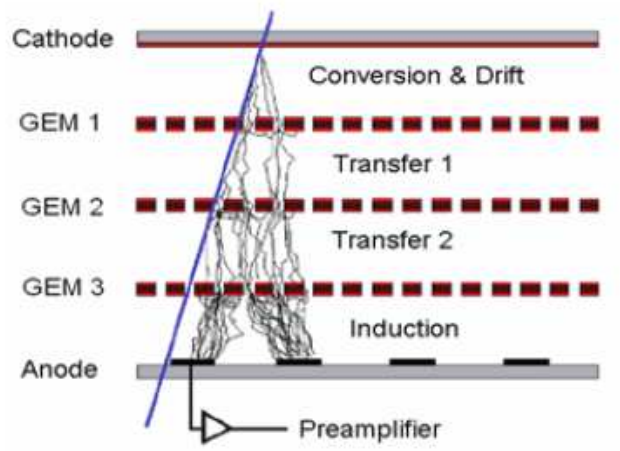

Fig. 1. Schematic diagram of the triple GEM detector.

The GEM technique allows separation of gas amplification and the readout electrode. For 1D position measurement the charge collecting strip electrode is an obvious scheme. Electric field in the induction gap between the last GEM and the readout strip plane (Fig. 1) enables control of the charge flow process. Efficient charge extraction towards the strips may be achieved with the proper field ratio in the induction gap and the GEM dipole. Additional advantage of such structure is that only fast electronic component of avalanches in gas drifts towards strips thus, the space charge effect due to positive ions is strongly reduced.

Charge diffusion in the whole amplification and collection chain is responsible for the broadening of the primary ionization spot when measured in the strip plane. If the diffusion is the only process changing the shape of the charge cloud during the drift (no strong distortion due to inhomogeneous electric field occurs) one can assume that the center of collected charge spot corresponds to the projection along electric field vector of the primary ionization center on the strip plane. Analysis of the cluster structure - pulse-heights recorded on the strip plane, allows the precise measurement of the primary ionization position. The output from each channel will be amplified and shaped by low noise charge sensitive amplifiers optimized for new generation 
gas detectors ( $\sim 10 \mathrm{~ns}$ rise time and $\sim 20 \mathrm{~ns}$ peaking-time). The signal from this amplifier will be sampled with a sampling rate of $\sim 125 \mathrm{MHz}$ (preliminary assumption) and digitized to reproduce the waveform. Cluster reconstruction, followed by cluster position analysis should allow on-line position reconstruction of single Xray hits [6]. X-ray energy will be estimated on the basis of the cluster total charge. Histograms will be constructed taking into account the energy tagging. In addition the corresponding software for current tokamak databases and all the diagnostics tools appropriate for data quality validation should be created and implemented into the detecting system. It includes a lot of various monitoring, communication, interface, data management tools etc. The algorithms and preliminary firmware preparation for digital data processing with $1 \mathrm{~ms}$ of time resolution is planned to be achieved during the conceptual design phase. The idea is to provide in real time a limited number of data at $1 \mathrm{kHz}$ rate (number of photon counts in 5 energy windows within $2-15 \mathrm{keV}$ of the whole energy range), whereas, in parallel, off-line storage of the full spectrum will be made at a rate of up to $10 \mathrm{kHz}$ to reach more precise analysis and obtain information about faster plasma phenomena. If needed and if photon statistics is sufficient faster rate can be programmed.

\subsection{GEM for WEST project}

\subsubsection{Conceptual design}

The WEST SXR system is comprised of two 1D cameras (vertical and horizontal views respectively), located in the same poloidal cross-section to allow for tomographic reconstruction. An array (about $20 \mathrm{~cm} \mathrm{x} 2$ $\mathrm{cm})$ consists of up to 128 detecting pixels in front of a $50 \mu \mathrm{m}$ width Beryllium pinhole, (equipped with a 0.1 $\mathrm{mm}$ diameter diaphragm) inserted in the case of the vertical camera at about $50 \mathrm{~cm}$ depth inside an optimized cooled thimble, see Fig. 2, in order to retrieve a wide plasma view. The horizontal camera remains outside the port, see Fig. 3.

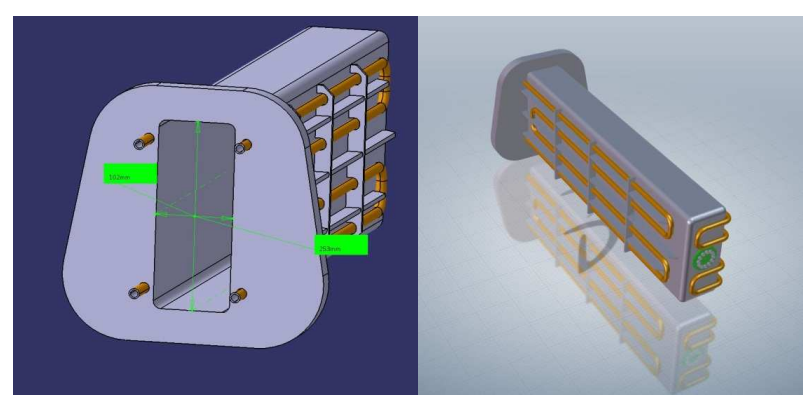

Fig. 2. Details about the cooled thimble (water).

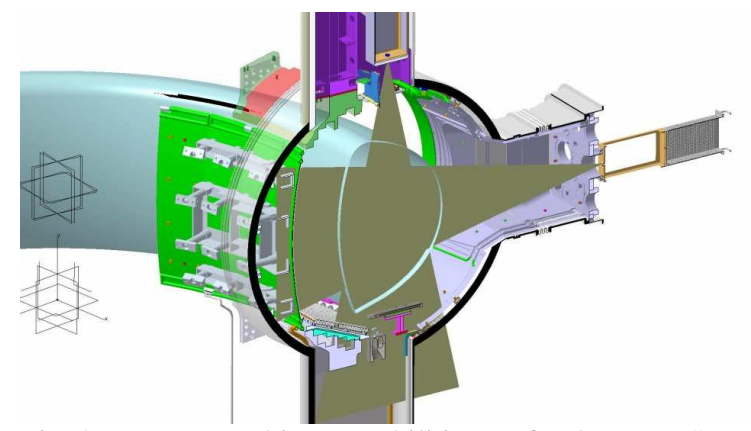

Fig. 3. Tomographic capabilities of the WEST SXR tomographic system based on GEM detectors. Up to 128 lines of sight per camera are possible. Vertical camera is inside the vertical port.

Acquisition of low energy spectrum is insured by a helium buffer installed between the pinhole and the detector. Complementary cooling systems (water) are used to maintain a constant temperature $\left(27^{\circ} \mathrm{C}\right)$ inside the thimble as can be seen in the schematic diagram in Fig. 4. The GEM detector operates at pressure slightly above the atmospheric one. A Beryllium window $(50 \mu \mathrm{m}$ thick) is used to maintain the vacuum inside the vessel and cut the very low part of the spectrum (less than 1 $\mathrm{keV})$.

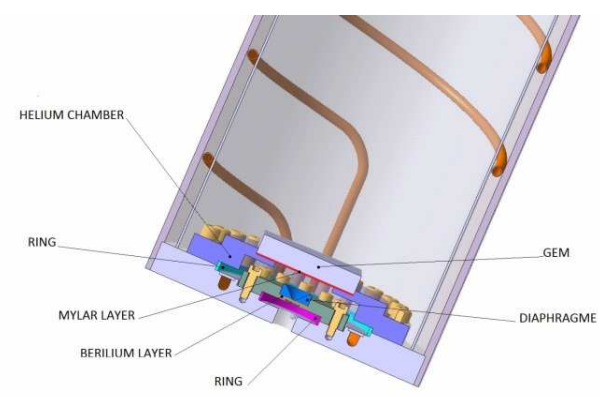

Fig. 4. Schematic principle of the cooled thimble associated to the helium buffer and GEM detector, beryllium window and diaphragm.

\subsubsection{Vertical camera positioning}

In order to position optimally the GEM camera inside the vertical thimble we simulated the magnetic field and temperature distribution inside its volume using the ANSYS code [7], Fig. 5 and 6.

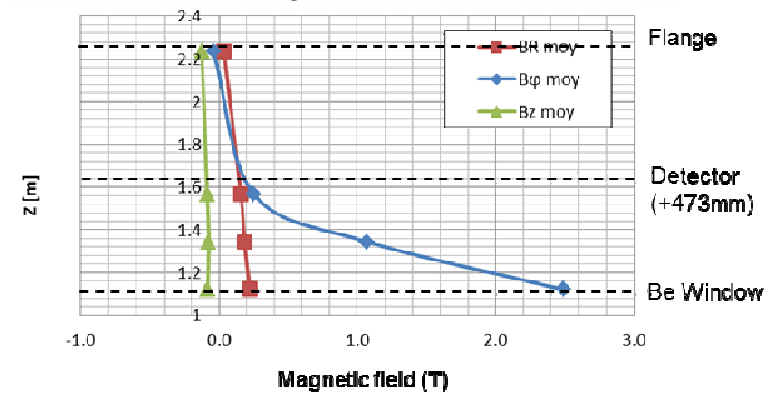

Fig. 5. Average magnetic field distribution within the thimble. 


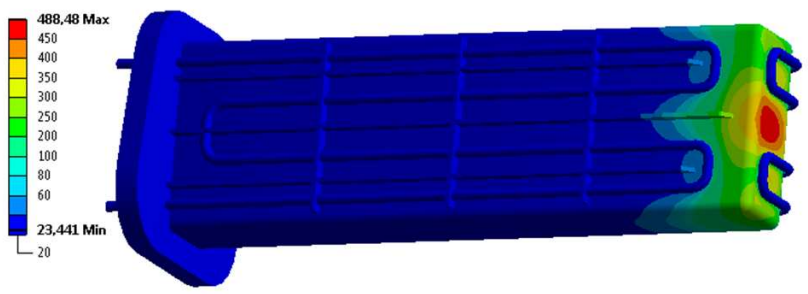

Fig. 6. Temperature distribution within the thimble.

Indeed the GEM detector cannot operate at temperature greater than $30^{\circ} \mathrm{C}$ and the magnetic field should be kept low if we want to be able to correct the deflection of the primary and secondary electrons induced by the magnetic field. The chosen WEST steady state configuration was an extreme case $(\mathrm{Ip}=1 \mathrm{MA}, \mathrm{BT}=4.3 \mathrm{~T})$ and the optimal position (related also to the space resolution we want to achieve in the equatorial plane) was found at $473 \mathrm{~mm}$ from the Be window. Concerning the thermal study a heat flux of $0,13 \mathrm{MW} / \mathrm{m} 2$ during $1000 \mathrm{~s}$ is considered on the lower face of the thimble. This flow corresponds to a power of 10MW uniformly distributed on the WEST plasma facing components. An external cooling of the thimble via exchanger providing water to room temperature $\left(\max .27^{\circ} \mathrm{C}\right)$ is considered. The flange is considered at $27^{\circ} \mathrm{C}$ (ambient temperature of the Torus). Perfect contact between the cooling and the casing is also considered. During a stationary discharge, the maximum temperature is of $490^{\circ} \mathrm{C}$ on the level of the Beryllium port-hole, see Fig. 6. Cooling is sufficient to decrease this temperature to room temperature $\left(27^{\circ} \mathrm{C}\right)$ on the level of the detector, $473 \mathrm{~mm}$ higher. A more precise study taking into account the Helium volume contained in the thimble shows that the conductivity of this last can be neglected. The temperature on the active surface of the detector is constant and equal to the temperature of cooling (difference of less than $0,05^{\circ} \mathrm{C}$ ), see Fig. 7 .
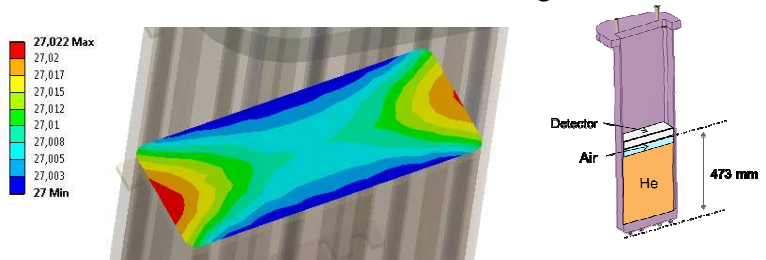

Fig. 7. (Left), temperature on the surface of the detector (10MW@1000s), taking into account the conductivity of the Helium buffer, (right) artistic representation of helium buffer and GEM detector positionning.

During the baking phases the port where diagnostics is located is at the temperature of $200^{\circ} \mathrm{C}$. The radiation on the thimble is then equivalent to a thermal power of 460W. Keeping active the cooling system, the temperature could then increase up to $50^{\circ} \mathrm{C}$ on the sides without pipes (and due to the lack of space could not be equipped). This is not acceptable for the diagnostic, that is why it is planned to withdraw the detector during the baking phases. A dedicated removable cassette containing the detector and the front-end electronics insuring pre amplification of the signal is foreseen for that purpose. On top of this in situ ${ }^{55} \mathrm{Fe}$ sources will be installed in front of the detecting part in order to insure a daily calibration of the diagnostics that could suffer from small pressure and/or temperature variations.

\section{Conclusions and perspectives}

The conceptual design of the GEM tomographic diagnostic for WEST has started. Preliminary simulations helped to position correctly the detector inside the vertical port of the machine associated to an innovative water cooling thimble and a Helium buffer to keep the capabilities to measure low energy emission. The horizontal camera will remain outside the port, allowing at the end an efficient tomographic diagnostics of the whole plasma section. The construction of the two final cameras is foreseen to be finished at the end of 2015. Preliminary tests with the prototype detector will be then performed on ASDEX Upgrade to optimize the detector design and associated algorithms. Then installation on WEST will start beginning of 2016, insuring SXR measurement during the first WEST plasma.

\section{Acknowledgments}

This project has received funding from the European Union's Horizon 2020 research and innovation programme under grant agreement number 633053. The views and opinions expressed herein do not necessarily reflect those of the European Commission. This scientific work was also partly supported by Polish Ministry of Science and Higher Education within the framework of the scientific financial resources in the year 2013 allocated for the realization of the international co-financed project.

\section{References}

[1] D. Vezinet, D. Mazon et al., Fast Nickel and Iron Density Estimation Using Soft X-Ray Measurements in Tore Supra: Preliminary Study, Fusion Science and Technology, 63(1), 9 (2013).

[2] D. Mazon et al., Soft X-ray tomography for real-time applications: present status at Tore Supra and possible future developments, Review of Scientific Instruments, 83(6), 063505 (2012).

[3] A. Bressan et al., High rate behavior and discharge limits in micro-pattern detectors, Nuclear Instruments and Methods A 424321 (1999).

[4] C. Altunbas et al., Construction, test and commissioning of the triple-gem tracking detector for compass, Nuclear Instruments and Methods in Physics Research A 490177 (2002).

[5] A. Kozlov et al., Development of a triple GEM UVphoton detector operated in pure CF4 for the PHENIX experiment, Nuclear Instruments and Methods in Physics Research A 523345 (2004).

[6] M. Chernyshova et al., Development of GEM gas detectors for X-ray crystal spectrometry, Journal of Instrumentation 9 C03003 (2014).

[7] C. Portafaix et al., 3D electromagnetic analyses during plasma disruption for the Tore Supra lower hybrid passive active multi-junction launcher, Proc. 26th SOFT conference, Porto (2016). 